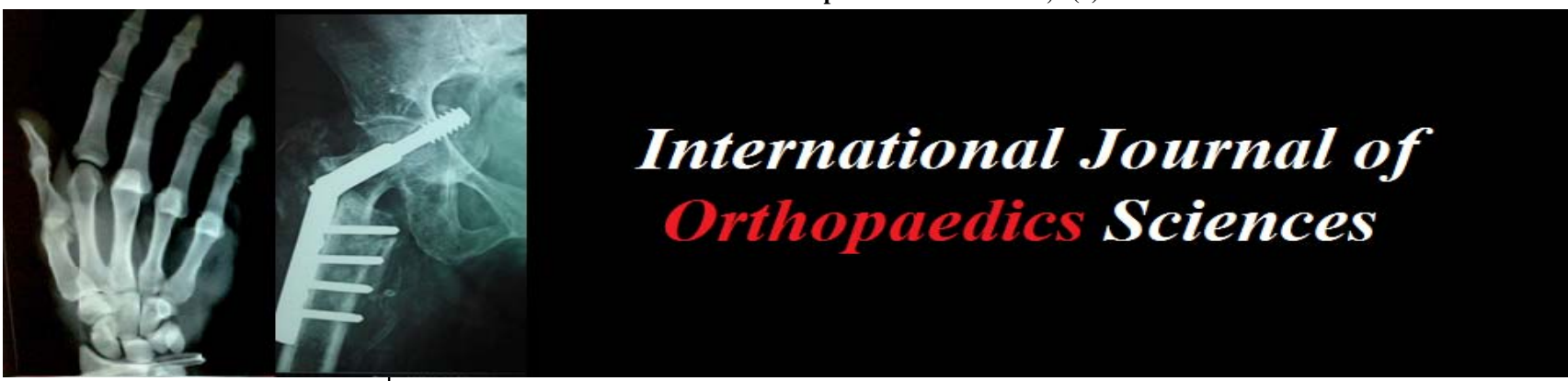

ISSN: 2395-1958

IJOS 2017; 3(3): 1086-1089

(C) 2017 IJOS

www.orthopaper.com

Received: 29-05-2017

Accepted: 30-06-2017

Dr. Sharad Hardikar

MB, FRCS, Head of Department

Hardikar Hospital, Pune,

Maharashtra, India

Dr. Shrinivas Prakash

MBBS, MS (Ortho)

Professor Hardikar Hospital,

Pune, Maharashtra, India

Dr. Madan Hardikar MBBS, MS (Ortho) Orthopedic surgeon Hardikar Hospital, Pune, Maharashtra, India

Dr. Saurabh Pagdal MBBS DNB resident Hardikar Hospital, Pune, Maharashtra, India

Correspondence Dr. Saurabh Pagdal MBBS DNB resident Hardikar Hospital, Pune, Maharashtra, India

\section{Clinical study of internal fixation of closed complex fractures of proximal humerus with single tine $T$ plate}

\author{
Dr. Sharad Hardikar, Dr. Shrinivas Prakash, Dr. Madan Hardikar and \\ Dr. Saurabh Pagdal
}

DOI: $\underline{\text { https://doi.org/10.22271/ortho.2017.v3.i3o.159 }}$

Abstract

Background: The management of complex proximal humerus fractures (Neer's type III, IV and fracture dislocation) is complicated. There are various treatment modalities available for proximal humerus fractures. We have designed and indigenously manufactured new plate called "SINGLE TINE PROXIMAL HUMERAL T-PLATE $(3.5 \mathrm{~mm})$ " for fractures of proximal humerus .The aim of this study was to examine the clinical outcome of complex proximal humeral fractures treated surgically with single tine T-plate.

Materials and Methods: This is retrospective observational study included 25 patients who underwent open reduction and internal fixation with single tine $\mathrm{T}$ plate for proximal humerus fractures in between 2010 and 2013. . All patients were evaluated using point system of Neer's shoulder evaluation system.

Results: Mean age of the patient 47.12 years (range 22-60 years). All the fractures united with a mean duration of 14 weeks (range 10 weeks to 18 weeks). As per Neer"s score we achieved excellent or satisfactory results in 21 out of 25 i.e. $84 \%$. The mean Neer"s score was 85.2 (range 64-94). We had unsatisfactory to poor result in 4 out of 25 cases (16\%). (Table 1) Complications were seen in 3 cases $(12 \%)$. One case of superficial wound infection and two cases of screw loosening.

Conclusion: Single tine T-plate is a good alternative implant for treating complex fracture and fracture dislocation of proximal humerus. It is cost effective, and easy to use. The single tine T-plate offers good support against varus mal-reduction. Primary bone grafting is a powerful tool in managing complex fractures/ fracture-dislocation of proximal humerus.

Keywords: Shoulder, humeral fractures, proximal, humerus head, dislocation fracture

\section{Introduction}

Proximal humeral fractures are the common fractures and represent about $4-5 \%$ of all fractures $[1,2,3]$. Although they may occur in any age group, an increased frequency occurs in older middle-aged and elderly individuals because of the age-related increase in osteoporosis ${ }^{[4,5]}$.

Majority of proximal humeral fractures can be treated conservatively ${ }^{[6,7]}$. But malunion is inevitable in conservative management ${ }^{[8,9]}$. In operative management, there are various types of fixation - transosseous suture fixation, percutaneous pinning, intramedullary nailing, or plate fixation ${ }^{[8,9]}$. They are used depends on the patient's age, activity level, and bone quality; the fracture type ${ }^{[8]}$.

Plate-and-screw constructs provide stable fixation for proximal humerus fractures ${ }^{[8]}$. Plates have undergone a series of modifications. Initial, T-Buttress Plates relied on proximal screws. Loss of reduction resulted when shoulder was mobilized because of TOGGLE between the screws \& plate and screw pull out in osteoporotic bones and comminuted fractures of proximal humerus ${ }^{[10]}$. Sehr \& Szabo ${ }^{[11]}$ bent the Semi-tubular Plate to form a blade plate. Later Hintermann ${ }^{12}$ designed a Cannulated Blade Plate. Currently locking plates are used widely. We have designed and indigenously manufactured new plate called "SINGLE TINE PROXIMAL HUMERAL T-PLATE $(3.5 \mathrm{~mm})$ )" for fractures of proximal humerus to prevent the problems like loosening of plate, varus collapse.

The aim of this study was to examine the clinical outcome of complex proximal humeral fractures treated surgically with single tine T-plate. 


\section{Materials and methods}

This is retrospective observational study included 25 patients (male 14and female 11) who underwent open reduction and internal fixation with single tine $\mathrm{T}$ plate for proximal humerus fractures in between 2010 and 2013.

Inclusion criteria included

1. Radiologically diagnosed cases with type III and type IV of the Neer classification ${ }^{[13]}$

2. Closed fractures

Exclusion criteria included

1. Neer's type 1 and 2 fractures

2. Compound fractures with any type.

3. Skeletally immature patients

\subsection{Operative method}

Preoperative true AP, lateral X-rays along with $3 \mathrm{~d}$ CT scan were done. Fracture patterns were classified according to the Neer classification ${ }^{[13]}$.

Surgery was performed in supine position using the deltopectoral approach.

Preliminary reduction of the fracture fragments is done under image guidance. In varus collapse of the head of humerus, a thick k-wire/ Steinmann pin is passed though the fracture into the medial aspect just below the inferior aspect of the humeral head and is used as a joystick to lift the humeral head and to correct the varus. Preliminary stabilization achieved using multiple $2 \mathrm{~mm} \mathrm{k}$-wires passed through the greater tuberosity quite proximally and across the lateral aspect of proximal humerus into the head so as to not to interfere with jig placement. Sometimes inter-fragmentary screws will be used to hold the fragments as well as to further ease the process of plate fixation. (Fig. 1)

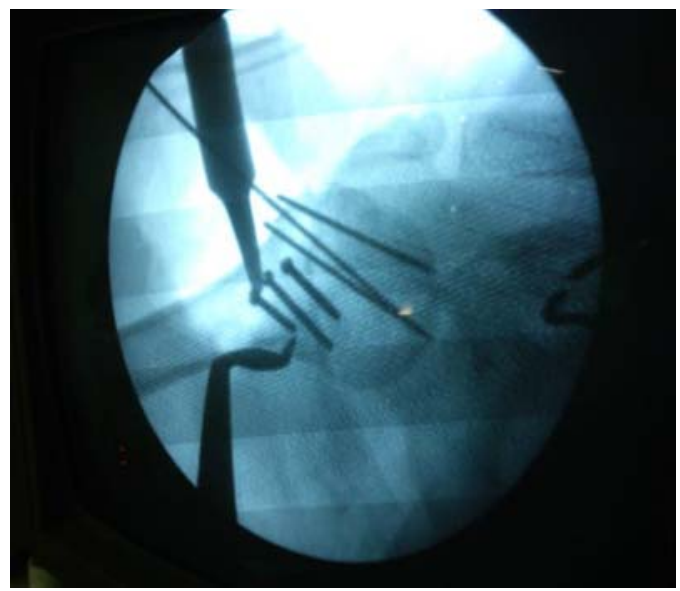

Fig 1: inter-fragmentary screws were used to hold fragments well.

In case of fracture dislocations, (A) If the lesser tuberosity is not fractured then two stay sutures are placed $2 \mathrm{~cm}$ proximal to the insertion of the subscapularis tendon. With limb in external rotation the subscapularis is then incised $1 \mathrm{~cm}$ proximal its insertion. Then the subscapularis tendon is retracted medially with Kocheres retractor and the dislocated head is delivered out and reduced into the glenoid cavity. Kwires are placed across the greater tuberosity and the proximal humerus to stabilize the humeral head. (B) If the lesser tuberosity is fractured then the tuberosity along with the tendon are both retracted medially to deliver the humeral head. The rest of the procedure is same as above.

Jig is placed over the lateral aspect of the humeral head taking care not to place is very high and its position is confirmed under image intensifier and $3.2 \mathrm{~mm}$ drill bit used to drill across the jig hole into the head up to the subchondral bone. Jig is removed and depth is measured by depth gauge and required length the tine is cut using a cutter. Tine is introduced into the hole created through the jig, gently hammering it until the vertical part is snug with the lateral cortex of the humerus. (Fig. 2)This plate is now secured with the bone using $3.5 \mathrm{~mm}$ cortical screws.

Single Tine $\mathbf{T}$ plate: Single tine T-plate (Fig. 3) is a modification of the T-buttress plate. It is a $3.5 \mathrm{~mm}$ plate with a single tine that protrudes from its proximal end. We used either 2X5 holed or 2X6 holed plate.

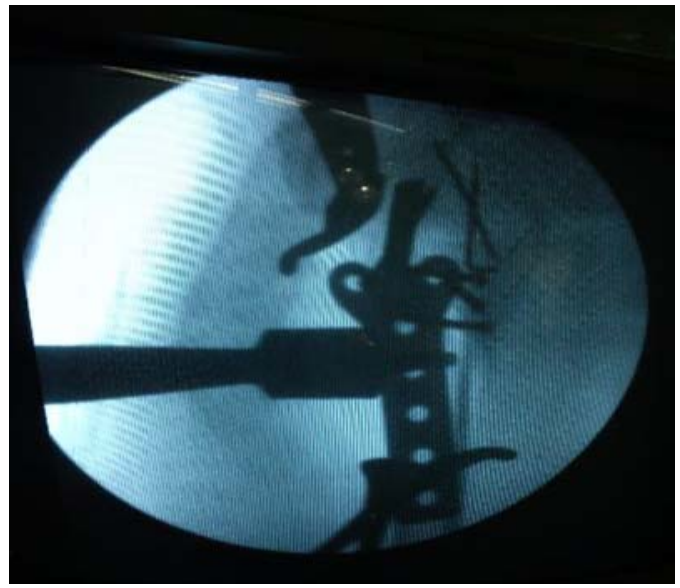

Fig 2: After using jig to make the entry point of tine, the tine of the t-plate is placed into the humeral head

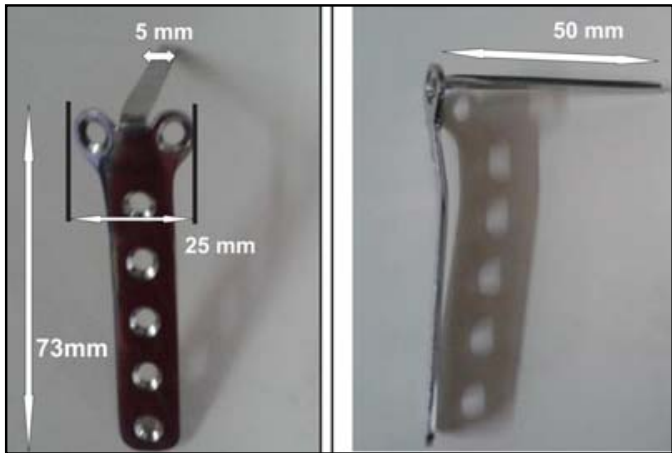

(a)

(b)

Fig 3: Single tine T-plate (5 holed) a) front view b) side view

2.2: The post operative rehabilitation: The post operative immobilization was done by chest arm strapping for 2 weeks. The post operative rehabilitation protocol included gentle pendulum exercise after 2 weeks and active shoulder mobilization with full range of motion after 4-6 weeks. Patients were followed up on OPD basis at 2, 4, 6,8,12, 24 weeks, 6th month, 12th month and at yearly interval. All patients were evaluated using point system of Neer "s shoulder evaluation system which is graded as Excellent, (above 90 units); satisfactory (80-89 units); unsatisfactory (70-79 units); failure (below 70 units). Analysis of the data done and the results will be expressed in terms of numbers and percentages.

\section{Results}

Mean age of the patient 47.12 years (range 22-60 years). All the fractures united with a mean duration of 14 weeks (range 
10 weeks to 18 weeks). As per Neer"es score we achieved excellent or satisfactory results in 21 out of 25 i.e. $84 \%$. The mean Neer "s score was 85.2 (range 64-94). We had unsatisfactory to poor result in 4 out of 25 cases $(16 \%)$. (Table 1) Complications were seen in 3 cases (12\%). One case of superficial wound infection and two cases of screw loosening.

Table 1: Grading of results by Neer's shoulder score

\begin{tabular}{|c|c|c|c|}
\hline Result & Units & No. of Cases & Percentage \\
\hline Excellent & more than 90 units & 9 & $36 \%$ \\
\hline Satisfactory & $80-89$ units & 12 & $48 \%$ \\
\hline Unsatisfactory & $70-79$ units & 2 & $8 \%$ \\
\hline Failure & less than 70 units & 2 & $8 \%$ \\
\hline
\end{tabular}

\section{Discussion}

Indications for operative treatment include displaced two-part surgical neck fractures, displaced $(>5 \mathrm{~mm})$ greater tuberosity fractures, displaced three-part fractures, and displaced fourpart fractures in young patients ${ }^{[8]}$. Plate-and-screw constructs provide the more stable fixation than transosseous suture fixation and percutaneous pinning ${ }^{[8]}$. Over the years, plates have undergone a series of modifications ${ }^{[11,12,14]}$. Locking plate gives good functional outcome especially in elderly and osteoporotic bone ${ }^{[3,8,15]}$.

Various studies of proximal humerus fracture osteosynthesis have reported various functional outcomes. Paavolainen $\mathrm{P}$ et $a^{[16]}$ in 1983 reported excellent or satisfactory results in 23 out of 31 patients $(74 \%)$. In another study by Wijgman et al [17] et al, they reported good to excellent results in $87 \%$ of their patients with three or four part proximal humeral fractures operated with a T-buttress plate and cerclage wires. Hintermann B et al ${ }^{[18]}$ used Blade plate in 38 patients with proximal humerus fractures, and reported good to excellent results in 30 patients $(78.94 \%)$, fair in 7 patients $(18.43 \%)$ and poor in 1 patient $(2.63 \%)$. Koukakis A et al ${ }^{[19]}$ studied 20 patients with proximal humeral fracture treated with Philos plate and the mean Constant score of this study was $76.1 \%$. Aggrawal $\mathrm{S}$ et al ${ }^{[3]}$ reported excellent to good satisfactory results in $55.5 \%$ cases of displaced proximal humerus fractures and poor outcome in $10.5 \%$ of the cases.

We have designed a special plate called fixed angle single tine proximal humeral plate. The fixed angled tine added for stability of proximal fracture fragment. T-plating and specific insertion of screws into the head (4 screws and tine in the head) gives us a good purchase of the proximal fragments i.e. head humerus. As per Neer's score, we achieved excellent or satisfactory results in 21 out of 25 i.e. $84 \%$. The mean Neer's score was 85.2 (range 64-94). (Fig. 4)

We used bone grafting in 10 cases. 3 cases (12\%) 3 part fracture and 3 cases (12\%) 4 part fracture and 4 cases $(16 \%)$ fracture dislocation. No bone grafting was done in 15 cases $(60 \%)$ of cases. We used autogenous bone graft from iliac crest or the proximal tibia in patients with poor bone quality, to fill up the fracture gaps between head, greater/lesser tuberosities, and shaft so as to give fracture stability.

In our series, 16 patients (64\%) had 3-part fracture type (Fig. 5). Four part fracture (Fig. 6) accounted for 16\% (4 patients) of total patients and 5 patients $(20 \%)$ had fracture dislocation. In our series both failure cases $(8 \%)$ occurred in four part fracture and fracture dislocation type. (Table 2). In the study by Aggrawal $\mathrm{S}$ et al ${ }^{[3]}$, five cases $(10 \%)$ of poor result occurred in AO-OTA Types B and C. In another study ${ }^{[15]}$, in cases with four-part fractures, the outcome was rated as excellent in $50 \%$, good in $22 \%$, fair in $13 \%$ and poor in $15 \%$. Complications were seen in 3 cases $(12 \%)$. We had one case of superficial infection noted on day 7th post op day. The culture revealed staph aureus organism. Oral antibiotic were given based on sensitivity and the wound healed. There were no cases of deep infection in our study. In first case of screw loosening, 56 years old female had screw loosening at 8 weeks follow-up. 12 weeks post-operative follow up radiograph showed fracture union (Fig. 7a). Only the loosened screw was removed at 12 weeks. Patient abduction improved to 120 degree by 16 weeks. In second case, 59 years old male had one screw loosening at 3 month post-operative follow up (Fig 7b). The loosened screw was removed.

Limitations in our study include small sample size, retrospective and non randomized study design.

Table 2: Functional outcome according to fracture types

\begin{tabular}{|c|c|c|c|c|c|}
\hline Type of fracture & No. of cases & Excellent & Satisfactory & Unsatisfactory & Failure \\
\hline Three part & 16 & 9 & 6 & 1 & 0 \\
\hline Four part & 4 & 0 & 2 & 1 & 1 \\
\hline Fracture dislocation & 5 & 0 & 4 & 0 & 1 \\
\hline Total cases & 25 & 9 & 12 & 2 & 2 \\
\hline
\end{tabular}

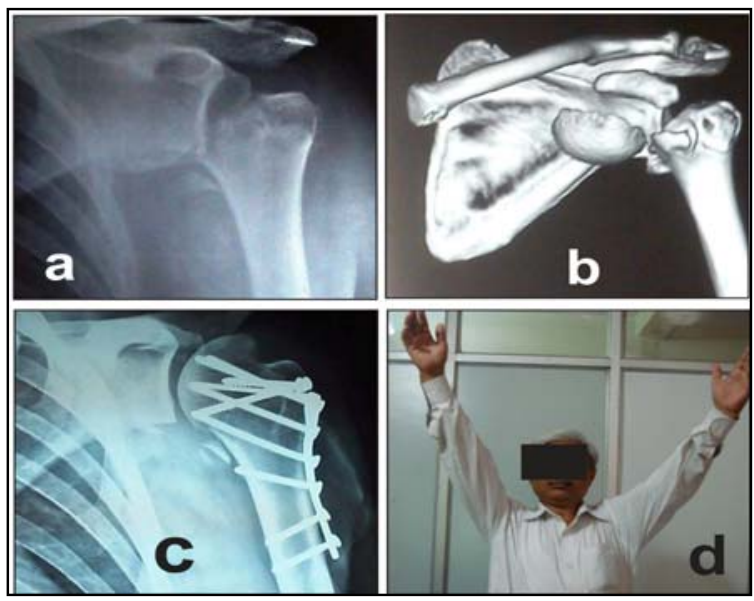

Fig 4: fracture dislocation type left proximal humerus fracture. a) Pre-operative x-ray b) Pre-operative 3d CT scan c) 1 year follow up x-ray d) abduction left shoulder up to $135^{\circ}$

$$
\sim 1088 \sim
$$




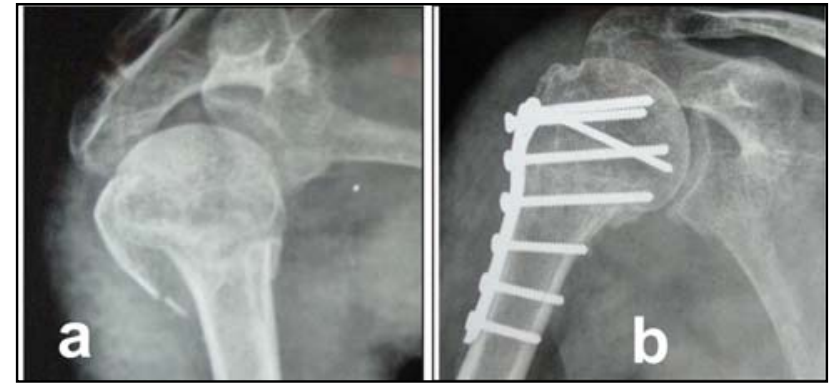

Fig 5: Neer's type III proximal humerus fracture. a) Pre-operative $x$ ray b) 1 year follow up $\mathrm{x}$-ray

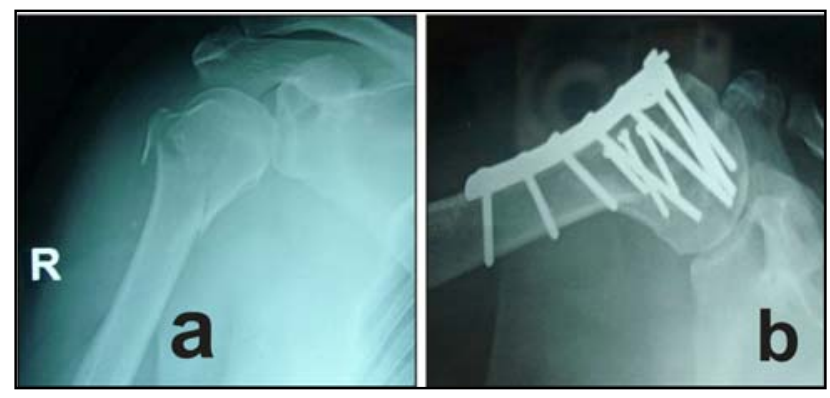

Fig 6: Neer's type IV proximal humerus fracture. a) Pre-operative $x$ ray b) 1 year follow up x-ray

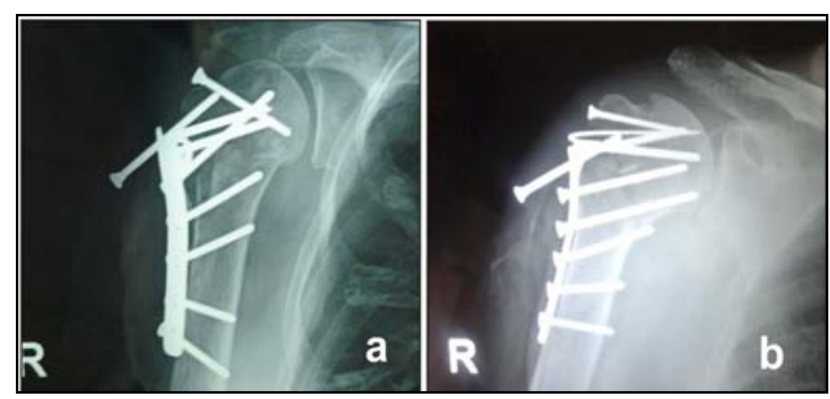

Fig 7a: first case of screw loosening in 56 year old female- 12 weeks post-operative follow up x-ray b) second case of screw loosening in 59 year old male- 12 weeks post-operative follow up x-ra

\section{Conclusion}

Single tine T-plate is a good alternative implant for treating complex fracture and fracture dislocation of proximal humerus .It is cost effective, and easy to use. The single tine T-plate offers good support against varus mal-reduction. Primary bone grafting is a powerful tool in managing complex fractures/ fracture-dislocation of proximal humerus.

\section{References}

1. Court-Brown CM, Garg A, McQueen MM. The epidemiology of proximal humeral fractures. Acta Orthop Scand. 2001; 72(4):365-71.

2. Calori, Giorgio Maria. Complications in proximal humeral fractures. Injury, 47, S54-S58.

3. Aggarwal S, Bali K, Dhillon MS, Kumar V, Mootha AK. Displaced proximal humeral fractures: an Indian experience with locking plates. J Ortho Surg Res. 2010; 5:60

4. Kim TI, Choi JH, Kim SH, Oh JH. The Adequacy of Diagnosis and Treatment for Osteoporosis in Patients with Proximal Humeral Fractures. Clinics in Orthopedic Surgery. 2016; 8(3):274-279.

5. Bergdahl C, Ekholm C, Wennergren D, Nilsson F, Möller M. Epidemiology and patho-anatomical pattern of 2,011 humeral fractures: data from the Swedish Fracture Register. BMC Musculoskeletal Disorders. 2016; 17:159.

6. Grubhofer, Florian. Reverse total shoulder arthroplasty for failed open reduction and internal fixation of fractures of the proximal humerus. Journal of Shoulder and Elbow Surgery, 26(1):92-100.

7. Jawa Andrew, Burnikel David. Treatment of Proximal Humeral Fractures: A Critical Analysis Review. JBJS Reviews. 2016, 4(1).

8. Perez EA. Fractures of the shoulder, arm, and forearm. In: Canale ST, Beaty JH. Campbell's Operative Orthopaedics. 12th Edition. Vol.3. Canada: Elsevier Mosby, 2012, 2841.

9. Robinson CM. Proximal humerus fractures. In Bucholz RW, Heckman JD, Court-Brown CM, Tornetta, editors: Rockwood and Green's fracture in adults, Philadelphia, Lippincott Williams \& Wilkins. 2010; 7:3 ${ }^{\text {rd }}$.

10. Kristiansen B, Christensen SW. Plate fixation of proximal humeral fractures. Acta Orthop Scand 1986; 57:320-3.

11. Sehr JR, Szabo RM. Semitubular blade plate for fixation of fractures of the proximal humerus. J Orthop Trauma. $1988 ; 2: 327-332$.

12. Hintermann B, Trouillier HH, Schafer D. Rigid internal fixation of the fractures of the proximal humerus in older patients. J Bone Joint Surg Br. 2000; 82:1107-1112.

13. Neer CS II. Displaced proximal humeral fractures: I. Classification and evaluation. J Bone Joint Surg Am. 1970; 52:1077-1089.

14. Esser RD. Treatment of three and four part fractures of the proximal humerus with a modified cloverleaf plate. $\mathrm{J}$ Orthop Trauma, 1994, 788-91.

15. Bigorre N, Talha A, Cronier P. A prospective study of a new locking plate for proximal humeral fracture. Injury. 2009; 40(2):192-6.

16. Paavolainen P, Bjorkenheim JM, Slatis P, Paukku P. Operative Treatment of Severe Proximal Humeral Fractures. Acta Orthop. Scand. 1983; 54:374-379.

17. Wijgman AJ, Roolker W, Patt TW. Open reduction and internal fixation of three and four-part fractures of the proximal part of the humerus. J Bone Joint Surg Am. 2002; 84:1919-25.

18. Hintermann B, Trouillier HH, Schafer D. Rigid internal fixation of the fractures of the proximal humerus in older patients. J Bone Joint Surg Br. 2000; 82:1107-1112.

19. Koukakis A, Apostolou CD, Taneja T. Fixation of proximal humerus fractures using the PHILOS plate: early experience. Clin Orthop Relat Res. 2006; 442:11520 . 